\title{
Harnessing the Inflammatory Reflex for the Treatment of Inflammation-Mediated Diseases
}

\author{
Yaakov A. Levine, Michael Faltys, and David Chernoff \\ SetPoint Medical, Inc., Valencia, California 91355 \\ Correspondence: ylevine@setpointmedical.com
}

\begin{abstract}
Treating diseases nonpharmacologically, using targeted neurostimulation instead of systemic drugs, is a hallmark of the burgeoning field of bioelectronic medicine. In this review, we provide a brief overview of the discovery and function of the prototypical neuroimmune reflex, the "inflammatory reflex." We discuss various biomarkers developed and used to translate early physiological discoveries into dosing parameters used in experimental settings, from the treatment of animal models of disease through a proof-of-concept clinical study in rheumatoid arthritis (RA). Finally, we relate how unique aspects of this form of therapy enabled the design of a next-generation implanted pulse generator using integrated electrodes, currently under evaluation in a U.S.-based clinical study for patients with drug refractory RA.
\end{abstract}

\begin{abstract}
$T^{\text {h }}$ he bioelectronic medicine application of using neurostimulation in immune and inflammatory disorders began as an effort to harness the "inflammatory reflex" to treat underlying chronic inflammatory processes. This treatment paradigm-shifting endeavor was based on foundational scientific discoveries of how the immune system is centrally monitored and modulated by the nervous system. To pioneer such a wide-open field, we took a methodical approach using objective immunologic biomarkers to empirically interrogate a range of electrical stimuli to produce the desired clinical outcomes. Such a tool was new to the field of neurostimulation, allowing us to quickly discover what the therapy required. This led us to not only a groundbreaking treatment approach, but enabled development of a novel neurostimulation device that was fundamentally different than any commercially available system. This review relates the
\end{abstract}

journey from concept to realization describing the process and discoveries made along the way.

\section{INFLAMMATORY REFLEX}

The inflammatory reflex is an innate physiological mechanism that the central nervous system uses to coordinate the immune response to infection, inflammation, or injury. The inflammatory reflex, a term coined by Dr. Kevin Tracey (Tracey 2002), encompasses both neural- and immune-cell components and is composed of two major axes. The first is afferent conveyance, within the vagus nerve to the brain, of neural information encoding detected peripheral inflammation. The second axis is the subsequent efferent response from the brain to the periphery through the motor fibers of the vagus nerve (Tracey 2002; Andersson and Tracey 2012a; Koopman 2017). The afferent arc of the reflex

Editors: Valentin A. Pavlov and Kevin J. Tracey

Additional Perspectives on Bioelectronic Medicine available at www.perspectivesinmedicine.org

Copyright (C) 2020 Cold Spring Harbor Laboratory Press; all rights reserved; doi: 10.1101/cshperspect.a034330

Cite this article as Cold Spring Harb Perspect Med 2020;10:a034330 
Y.A. Levine et al.

was identified by Nijiima in the mid-1990s, who showed that sensory activity within the vagus nerve increased dramatically on intraperitoneal (IP) injection of interleukin (IL)-1 $\beta$ (Niijima 1996), and by Linda Watkins and colleagues, who showed that an intact vagus nerve was necessary and sufficient to enable the central fever response to IP IL-1 $\beta$ (Watkins et al. 1995). A few years later, the efferent "motor" arm was first shown in animal models of acute inflammation by Tracey and colleagues (Borovikova et al. 2000). Activity of the efferent vagus nerve initiated by activation of vagal motor nuclei resulted in reduced systemic production of proinflammatory cytokines. This effect was later shown to be dependent on binding of acetylcholine $(\mathrm{ACh})$ to the nicotinic acetylcholine receptor type 7 ( $\alpha 7 \mathrm{nAChR})$ on cytokine-producing immunocytes (Wang et al. 2003; Parrish et al. 2008). This motor pathway has been referred to as the cholinergic anti-inflammatory pathway (CAP) (Fig. 1; Tracey 2002).

Numerous publications from laboratories worldwide have since provided additional details of the anatomic, cellular, and molecular pathways involved in the inflammatory reflex (Fig. 1 ), as well as supporting evidence of central control over aspects of inflammation through these neuroimmune pathways, reviewed elsewhere (Andersson and Tracey 2012b; Olofsson et al. 2012b; Matteoli and Boeckxstaens 2013; Levine et al. 2018a; Pavlov et al. 2018). These abundant and varied reports have converged on two major findings. The first is that activation of the inflammatory reflex, either by electrical vagus nerve stimulation (VNS) or the administration of nicotine or specific $\alpha 7 \mathrm{nAChR}$ agonists, can decrease the signs and symptoms of inflammation-driven disease in disparate models. The second, conversely, is that disrupting the innate tonic control of inflammation through physically severing or chemically denervating the vagus or splenic nerves, or through disruption of the $\alpha 7 \mathrm{nAChR}$ by genetic manipulation or chemical antagonism, can increase the inflammatory disease burden (Fig. 2). Recent evidence has emerged linking VNS to the modulation of specialized proresolving mediators (SPMs), a class of bioactive metabolites of essential fatty acids that play a central role in the resolution of inflammation, distinct from immunosuppression (Serhan 2014). SPMs, which have been isolated from both human and rodent vagus nerves, are similarly increased following VNS or ACh administration, reducing proinflammatory neutrophil infiltration while simultaneously enhancing macro-

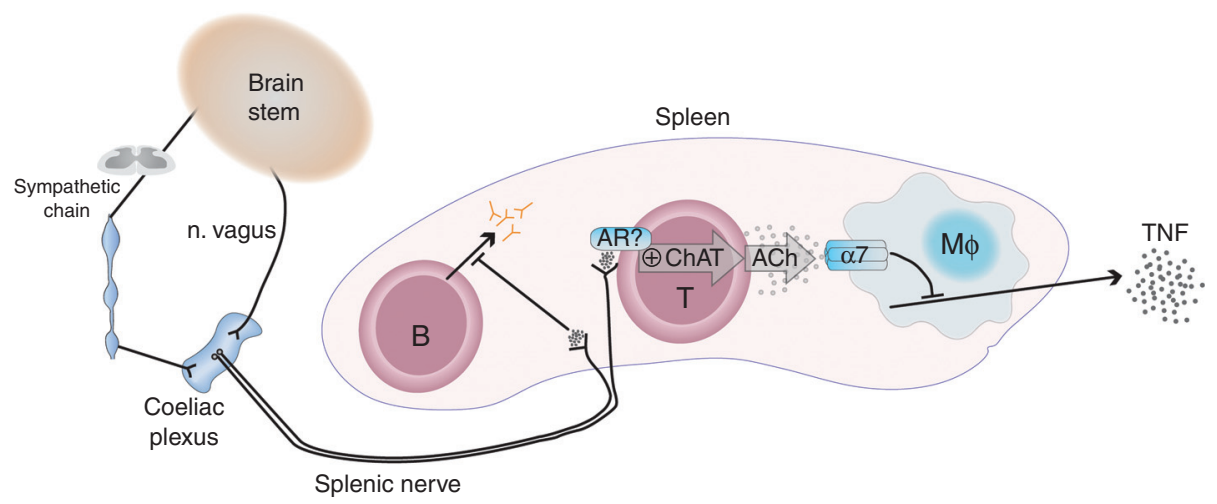

Figure 1. Model of the cholinergic anti-inflammatory pathway (CAP), the efferent arc of the inflammatory reflex. Signals from the brainstem travel through the vagus nerve to the celiac plexus, which also receives input from the sympathetic trunk. The splenic nerve arises in the coeliac plexus and projects to the spleen. Choline acetyltransferase (ChAT)-expressing $\mathrm{T}$ and $\mathrm{B}$ cells are found in proximity of splenic nerve terminals. Motor signals in the vagus nerve activate the splenic nerve, which releases norepinephrine, activating ChAT $+\mathrm{T}$ cells through adrenergic receptors (ARs), and induces the production and release of T-cell-derived acetylcholine (ACh). This ACh then acts on the $\alpha 7$ nicotinic ACh receptors on macrophages and other immune cells and suppresses release of the tumor necrosis factor (TNF). (Reprinted, with permission, from Olofsson et al. 2012b, (c) Wiley.) 


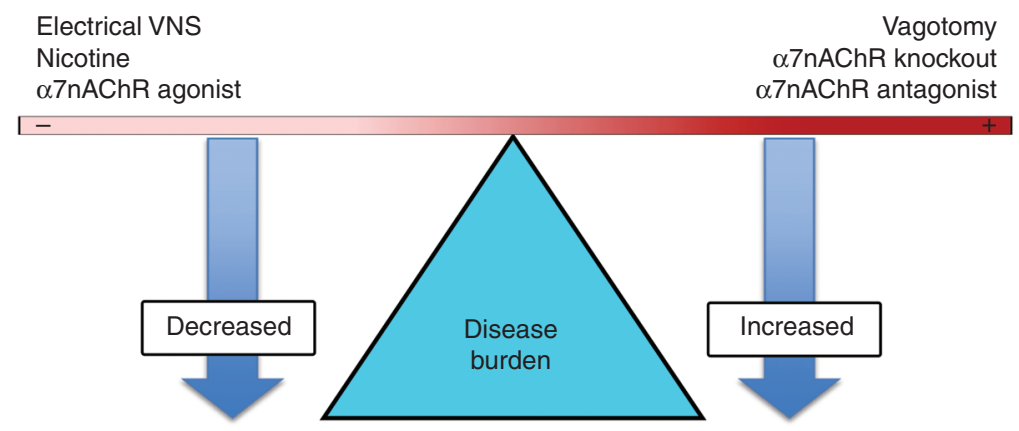

Figure 2. Experimental manipulation of the cholinergic inflammatory pathway influences disease burden in preclinical models of inflammation-driven disease. Electrical and pharmacologic stimulation of the cholinergic anti-inflammatory pathway (CAP) decreases, whereas surgical, genetic, or pharmacologic disruption of the CAP increases disease burden in preclinical models of rheumatoid arthritis (RA) and inflammatory bowel disease (IBD). $\alpha 7 \mathrm{nAChR}$, Nicotinic acetylcholine receptor type 7; VNS, vagus nerve stimulation. (Reprinted, with permission, from Levine et al. 2014a.)

phage bacterial clearance (Dalli et al. 2017; Serhan et al. 2018). Conversely, SPM levels are decreased following vagotomy, associated with greater inflammation and reduced bacterial clearance (Mirakaj et al. 2014; Dalli et al. 2017).

Whereas the fundamental discoveries described a novel mechanism for central control over inflammation and immunity, they also established that the reflex could be harnessed by electrical stimulation of the vagus nerve to therapeutically modulate disease in animal models driven by inflammation. These included several rodent models of rheumatoid arthritis (RA) and inflammatory bowel disease (IBD) (Meregnani et al. 2011; Sun et al. 2013; Levine et al. 2014a, 2018a), both of which have a large unmet clinical need for new treatments that do not compromise host response to infection. The promise of developing a nonpharmacologic and potentially much safer treatment option for the millions of patients suffering from debilitating chronic conditions provided the impetus for translating these discoveries in animals to the clinical-dosing parameters and novel technologies currently under evaluation in a first-in-human bioelectronic therapy trial.

\section{BIOMARKERS}

To develop a neuromodulation therapy based on manipulating the inflammatory reflex in chronic inflammatory disease, one first must understand the scope of physiologic responses to a range of exogenous electrical interventions. The most expedient way of accomplishing this is through the use of biomarkers, which has been defined by the National Institutes of Health (NIH) health biomarkers working group as "a characteristic that is objectively measured and evaluated as an indicator of normal biological processes, pathogenic processes, or pharmacologic responses to a therapeutic intervention" (Biomarkers Definitions Working Group 2001). The optimal biomarker(s) for tracking the effect of a neuromodulation application need to be measurable in clinically accessible samples and rapidly adaptive to changes in treatment parameters, as well as highly correlated to the intended effect of the treatment. Exemplars include the activation of a muscle group in response to motor nerve stimulation or the suppression of Parkinsonian tremor following deep brain stimulation.

The simplest biomarker to measure for VNS bioactivity is a change in heart rate. Because the vagus nerve innervates the cardiac pacemaker at the sinoatrial node, changes in vagus nerve activity can affect heart rate. Depending on the condition, VNS can either increase or decrease the heart rate (Ardell et al. 2017). Heart rate changes can indicate coactivation of specific fibers within the vagus that carry anti-inflammatory signaling. However, the vagus nerve is a 
Y.A. Levine et al.

highly heterogeneous conduit composed of many different types of neurons with various coordinated, yet mostly independent functions. Therefore, various outcomes of vagus nerve activities are dissociable and potentially divergent. For example, heart rate changes can be measured without activation of the inflammatory reflex, and, conversely, modulation of cytokines can occur without detectable changes to heart rate. Therefore, measurement of heart rate is often not appropriate for tracking manipulation of the inflammatory reflex. A more complete reflection of autonomic nervous system activity, composed of vagus nerve and sympathetic tone and their balance, may be measured by recording cardiac $\mathrm{R}-\mathrm{R}$ intervals (heart rate variability [HRV]) and then performing power spectral analyses on the data. These types of HRV analyses have shown correlations between reduced vagus nerve tone with serum c-reactive protein levels and disease activity in RA (Evrengul 2004; Sajadieh et al. 2004; Koopman et al. 2016b). Although not yet validated as a tool for monitoring the activity of the inflammatory reflex specifically, the potential of HRV as a biomarker for closed-loop stimulation of the inflammatory reflex has been discussed elsewhere (Levine et al. 2017).

The rodent endotoxemia model was used in many experiments to explore the effects of VNS on systemic inflammation. In those studies, endotoxin, or lipopolysaccharide (LPS), a cell wall component of gram-negative bacteria, was administered centrally, either IP or intravenous (IV), and serum tumor necrosis factor $\alpha$ (TNF- $\alpha$ ) levels or animal survival were measured as outcome biomarkers. Circulating TNF- $\alpha$ in endotoxemia is predominantly released by splenic monocytes/macrophages, as removal of the spleen reduced TNF production by $80 \%$ (Huston et al. 2006). However, endotoxemia has several drawbacks that limit its use as a biomarker for translational studies. First, it is rarely appropriate for time course experiments, because the exogenous toxin causes a systemic reaction, which in high doses is injurious or lethal and in low doses alters the baseline responsivity of the organism to a subsequent challenge (He et al. 1992). Second, this systemic challenge may affect the course of any disease model tested. Third, endotoxin injection/infusion causes systemic symptoms (Konsman et al. 2002), which makes it difficult to justify its use in large mammals and humans.

To overcome these drawbacks when confirming that the inflammatory reflex has been activated, we and others developed ex vivo bioassays in which blood is sampled and challenged with endotoxin. In principle, the measurement of monocyte responsiveness to an endotoxin challenge captures a snapshot of the immunological "set point" of these circulating leukocytes, which relates to how these cells respond to infection or sterile injury. The ex vivo bioassay allows for multiple sequential and independent tests that can measure changes in the inflammatory phenotype of circulating leukocytes over time or across therapies. In addition, the ex vivo bioassay allows for LPS dose ranges that provide a broader understanding of physiologic responses. The whole blood ex vivo bioassay has been developed and adapted for humans and large animals, rats, and mice. A major limitation of this bioassay is the removal of the central nervous system and other cells and systems that may interact with the circulating cells. A second limitation is the time and special equipment required to standardize performance of a low throughput bioassay used to measure the concentration of relevant analytes within the supernatant.

\section{STIMULATION PARAMETERS}

To design therapeutic electrical dosages that will eventually be administered to patients with disease, an understanding of the relationship between stimulation parameters and the immune-modulating end effect must be developed. Stimulation waveform parameters, such as output current and frequency, are among the key determinants of functional stimulation other than duty cycle, stimulation location, and electrode design, discussed below.

\section{Output Current}

The output current intensity and charge density are critical determinants of the neural fibers that 
are activated and thus the downstream effects of stimulation. As charge (charge = current intensity $\times$ pulse width) is injected into the nerve, the axonal transmembrane potential is altered. An action potential is generated once the transmembrane potential reaches a critical threshold. Early work performed by the Tracey group and others used constant voltage stimulation to show TNF modulation in rodents, often in the range of 1-5 V (Borovikova et al. 2000; Huston et al. 2006; Rosas-Ballina et al. 2008, 2011; Vida et al. 2011). However, when stimulating with constant voltage, the amount of current driven through the nerve is highly dependent on various electrical resistances in the circuit, including electrode materials and geometry as well as electrode contact with tissue. Although somewhat controllable in the laboratory, it is well-known that impedances of implanted electrodes change over time because of tissue remodeling and electrode surface electrochemical changes. To translate the early discoveries into a consistent therapy, we first methodically investigated a range of constant current stimulus parameters in rodents. Keeping pulse width and frequency constant at $0.2 \mathrm{msec}$ and $10 \mathrm{~Hz}$, respectively, output current intensity was increased until the suppression of LPS-induced TNF production became evident, above the "threshold current" (Olofsson et al. 2015). The empirically determined threshold current was between 0.25 and $0.50 \mathrm{~mA}$ in the mouse and between 0.10 and $0.25 \mathrm{~mA}$ in the rat. The absolute difference between the two species was likely related to the different electrode geometries used in the experiments. Important for stimulation design, there was no significant loss-of-function observed across the range of intensities above the threshold current. Many subsequent rodent studies were performed using constant current at 0.75$1 \mathrm{~mA}$ or even 2-3 $\mathrm{mA}$, to ensure the therapeutic threshold was exceeded (Costantini et al. 2010; Olofsson et al. 2012a, 2015; Levine et al. 2014b; Lu et al. 2014; Li et al. 2016).

Identification of the specific neuron class that transmits the effector signal can improve the design of therapeutic stimulation. To identify the neuronal fibers within the estimated 100,000 neurons within the cervical vagus nerve
(Hoffman and Schnitzlein 1961), we performed neurophysiologic recording on the rat vagus nerve over a wide range of current intensities. Verified with compound action potentials measured in the abdomen, a set of transdiaphragmatic myelinated fibers were activated at the same threshold current as TNF suppression, indicating that depolarizing these neurons is sufficient to initiate the inflammatory reflex and reduce LPS-induced TNF (Olofsson et al. 2015). To characterize the excitation properties of the vagus nerve in large mammals, Grill and colleagues performed a series of neurophysiology experiments in canines (Castoro et al. 2011; Yoo et al. 2013). Stimulation intensity inputoutput curves were constructed, which indicated that myelinated fibers begin to be recruited at 0.4-0.5 mA. In a set of independent experiments, we have found recruitment of these myelinated fibers as low as $0.25 \mathrm{~mA}$ using a stimulating electrode design that has been shown to allow more efficient delivery of current through the nerve relative to the open helical electrodes commonly used for VNS (Levine et al. 2018b). Analogous to the functional experiment in rats described above, modulation of ex vivo LPS-induced TNF was first detected at $0.25 \mathrm{~mA}$ in canines (Y Levine, unpubl.).

Current clinical devices placed on the cervical vagus nerve have labeled upper limits for output current of $3.5 \mathrm{~mA}$, although currents as high as $5.5 \mathrm{~mA}$ have been used in clinical studies of VNS to treat heart failure (De Ferrari et al. 2011; Gold et al. 2016). In the controlled pivotal clinical studies in drug-resistant epilepsy, current was up-titrated as tolerated to a maximum of $3.5 \mathrm{~mA}$, but maximal average output current tolerated by the subjects was actually about 1.3 mA (Handforth et al. 1998). An early summary of clinical experience with device parameters notes that output currents above $2 \mathrm{~mA}$ are "rarely necessary and can reduce patient tolerability" (Heck et al. 2002). To date, there have been four clinical trials investigating direct VNS to treat RA (ClinicalTrials.gov identifiers: NCT015529 41, NCT03437473) and Crohn's disease (ClinicalTrials.gov identifiers: NCT01569503, NCT02 311660). In the two studies that have published outcomes, subjects were implanted with Cyber- 
Y.A. Levine et al.

onics (now Livanova) VNS systems that are clinically approved for drug-resistant epilepsy and depression. The subjects were initially stimulated at $0.25 \mathrm{~mA}$ and then gradually up-titrated as tolerated to a maximum output of $1.25 \mathrm{~mA}$ (Bonaz et al. 2016) or $2.0 \mathrm{~mA}$ (Koopman et al. 2016a), resulting in dramatic improvement in clinical scores in each respective study.

\section{Pulse Width and Frequency}

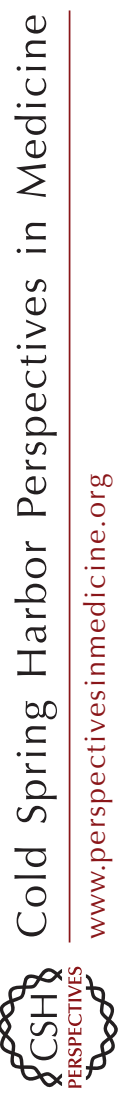

Along with current intensity, pulse width is another integral determinant of neuronal activation. The interaction between these two parameters is known as the strength-duration relationship. The threshold current required for activation decreases as the pulse width increases. This relationship has been modeled mathematically as either the Lapique or Weiss equation, and can be solved around two neuron/electrode-specific variables, the rheobase and the chronaxie (McIntyre and Grill 1998). The rheobase is defined as the minimum current amplitude necessary to depolarize the neuron given a pulse width of infinite length (an asymptotic region within the mathematical models), and the chronaxie is defined as the pulse width required to depolarize the neuron given a current intensity twice the rheobase. Stimulating with a pulse width equal to the chronaxie is more charge-efficient than stimulating at longer pulse widths, limiting damage to the tissue and electrode and preserving battery life of a pulse generator. As mentioned above, Grill and colleagues performed a series of vagus nerve neurophysiology experiments in canines and determined empirically that the chronaxies of both $\mathrm{A}$ and $B$ myelinated fibers were about $0.2 \mathrm{msec}$ (Castoro et al. 2011). These findings indicate that the most efficient pulse width to activate the myelinated fibers within the vagus nerve will be about $0.2 \mathrm{msec}$.

Consistent with this finding, early work by Tracey and colleagues exploring VNS in endotoxemia found no significant difference in the extent of serum TNF modulation between pulse widths of 0.5 and $2.0 \mathrm{msec}$ (Huston et al. 2007). In addition, they reported no difference in serum TNF modulation when stimulating at $5 \mathrm{~Hz}$ ver- sus $30 \mathrm{~Hz}$; however, there was a greater reduction in heart rate at the higher frequency, as would be predicted by Ardell (Ardell et al. 2017) and others (Heck et al. 2002). Our laboratory subsequently showed no significant difference in serum TNF modulation in endotoxemic mice when stimulated at frequencies between 1 and $10 \mathrm{~Hz}$ (Olofsson et al. 2015). However, there may be contexts in which frequency dependence becomes apparent, as there appears to be stimulation frequency-dependent norepinephrine release in the spleen that peaks at $10 \mathrm{~Hz}$ (Kees et al. 2003), potentially improving cytokine modulation. Furthermore, there was a recent report by Boeckxstaens and colleagues indicating a differential effect on IL-6 and IL-8 (but not TNF) levels in ex vivo LPS-stimulated whole blood of patients following acute posterior subdiaphragmatic vagus stimulation at $5 \mathrm{~Hz}$ versus $20 \mathrm{~Hz}$ (Stakenborg et al. 2017). Additional work by our group in rodent endotoxemia (mouse and rat), rat collagen-induced arthritis, rat indomethacin-induced intestinal inflammation, and canine ex vivo LPS-stimulated whole blood assay models showed that stimulating the vagus nerve with a pulse width of $0.2-0.25 \mathrm{msec}$ and pulse frequency of $10 \mathrm{~Hz}$ was effective at modulating cytokines and reducing signs and symptoms of disease (Levine et al. 2013, 2014b; Olofsson et al. 2015; Y Levine, unpubl.). Many other laboratories have also reported an immune modulatory effect when stimulating the vagus nerve at pulse widths between 0.2 and $2 \mathrm{msec}$ and frequencies between 1 and $20 \mathrm{~Hz}$, reviewed by Kwan et al. (2016).

It has been reported in the clinical epilepsy literature that pulse width strongly affects the tolerability of VNS in clinical studies with significant improvements in tolerability as pulse duration is lowered from 0.5 to $0.25 \mathrm{msec}$ (Heck et al. 2002). Typical clinical pulse frequencies for treatment of epilepsy and depression range between 20 and $30 \mathrm{~Hz}$. In the two successful published clinical studies of VNS to treat RA and Crohn's disease, subjects were stimulated with a pulse width of $0.25 \mathrm{msec}$ and frequency of $10 \mathrm{~Hz}$ (Koopman et al. 2016a) or a pulse width of $0.50 \mathrm{msec}$ and frequency of $20 \mathrm{~Hz}$ (Bonaz et al. 2016). 
Harnessing the Inflammatory Reflex for Treatment of Disease

\section{Duty Cycle}

In the context of neuromodulation, duty cycle describes the amount of time that the stimulator is on relative to total time off; the two major components of this parameter being the pulse train length and the intertrain interval. To explore the relationship between pulse train length and efficacy, Tracey and colleagues varied VNS pulse train length in murine endotoxemia and reported no difference in the extent of TNF modulation following $30 \mathrm{sec}$ of VNS versus VNS pulse trains as long as $20 \mathrm{~min}$ (Huston et al. 2007). However, the interindividual variance was greater at $30 \mathrm{sec}$ than at $60 \mathrm{sec}$ or longer, so most of the studies performed by our group included pulse trains of at least $60 \mathrm{sec}$. This pulse train length dosing observation was later extended to find the shortest effective pulse train in murine endotoxemia, which was unexpectedly and unprecedentedly a single pulse (Olofsson et al. 2015). To explain this extremely low trigger for the systemic anti-inflammatory effect, we hypothesized that a single suprathreshold pulse can trigger a feed-forward amplification within the spleen, as evidenced by the increasing levels of ACh release in the spleen for tens of minutes following VNS in mice (RosasBallina et al. 2011).

One unique and striking aspect of the inflammatory reflex is its long-lasting anti-inflammatory effect following induction, as observed in cultured cells, mice, rats, canines, and humans. Primary human macrophages in culture were treated with ACh for $1 \mathrm{~h}$, followed by an endotoxin challenge for $4 \mathrm{~h}$ in fresh media. Levels of LPS-induced TNF were significantly reduced from sham-treated cells at 2, 24, and 48 h postACh exposure, but not at $72 \mathrm{~h}$ (Huston et al. 2007). A similar physiologic half-life was observed in vivo in murine endotoxemia. Mice exposed to $0.5 \mathrm{~min}$ of VNS on day 0 were challenged at various times thereafter with IP endotoxin, and serum TNF was measured $90 \mathrm{~min}$ later. The investigators reported that the reduction in serum TNF at $24 \mathrm{~h}$ was similar to the reduction at $2 \mathrm{~h}$ post-VNS. The reduction in serum TNF at $48 \mathrm{~h}$ was still significantly reduced as compared to sham VNS, but no longer at $72 \mathrm{~h}$
post-VNS (Huston et al. 2007). Various molecular mechanisms underlying the long-term effect of VNS are still under investigation. Key dependencies of the effect on cells of macrophage lineage have recently been identified on adenylyl cyclase 6 and the phosphorylation of cAMP response element binding protein (Tarnawski et al. 2018).

Similar to the long-term suppression in TNF in rodents, TNF release in LPS-stimulated whole blood was significantly reduced from baseline for at least $48 \mathrm{~h}$ following $60 \mathrm{sec}$ of VNS in chronically implanted canines (Y Levine, unpubl.). To confirm the long-lasting effect on TNF production is conserved in humans, otherwise healthy subjects with epilepsy underwent implantation of a VNS system that included an intraoperative stimulation. Compared to the LPS-induced TNF levels in the ex vivo bioassay pre-VNS, the TNF levels at $4 \mathrm{~h}$ post-VNS were significantly reduced (Koopman et al. 2016a).

In the clinical treatment of drug refractory epilepsy and depression, duty cycles ranging from $10 \%$ to $40 \%$ are typically used. In acute endotoxemia models in rats and in vitro whole blood assay stimulation experiments in dogs, stimulation periods of 1-2 min were optimal for suppression of LPS-induced TNF production. Extension of stimulation times up to 20 min did not confer additional efficacy in rodent endotoxemia, and stimulation for 1 min daily in the rat collagen-induced arthritis model was sufficient to inhibit disease (Levine et al. 2014b). In the two successful, published clinical studies of VNS to treat RA and Crohn's disease, subjects were stimulated with duty cycles of $0.07 \%-0.28 \%$ (1-4 min/day) (Koopman et al. 2016a) or $10 \%$ (Bonaz et al. 2016).

\section{STIMULATION LOCATION AND ELECTRODE ARCHITECTURES}

As the nature of the inflammatory reflex was developed and validated and the therapeutic potential became clear, we and others began to explore alternative ways and physical access points to target with electrical stimulation. To compete with direct stimulation on the cervical vagus nerve and develop a successful clinical 
Y.A. Levine et al.

intervention, the electrode placement must consistently activate the nerve and reflex with results comparable to direct stimulation of the vagus nerve. Because the splenic nerve and spleen are downstream from the cervical vagus nerve, groups have stimulated the splenic nerve to modulate circulating cytokines in isolated organ perfusion (Kees et al. 2003) and rodent endotoxemia (Levine et al. 2013) demonstrating TNF- $\alpha$ modulation. Splenic nerve stimulation was performed acutely in a pig model of endotoxemia, resulting in attenuated hypotensive shock and serum TNF- $\alpha$ (Shafer 2010). To explore the feasibility of a chronic splenic nerve solution, our group implanted cuff electrodes onto the splenic nerve in a porcine model. However, we encountered surgical complications that convinced us that the splenic nerve would be a far more difficult target than the vagus nerve (M Faltys, unpubl.). The subdiaphragmatic vagus nerve has also been targeted for modulating inflammation, primarily to prevent postoperative ileus, as the nerve is readily accessible during surgical laparotomies. Boeckxstaens and colleagues have reported that both cervical as well as subdiaphragmatic acute VNS reduce the inflammatory and intestinal motility sequalae of ileus (Matteoli et al. 2014; Stakenborg et al. 2017).

There are a few groups that have reported immune-modulating and autonomic activity following transcutaneous electrical stimulation of the auricular branch of the vagus nerve ( $\mathrm{Gao}$ et al. 2008; Zhao et al. 2012). In one study, endotoxemic rats were subjected to either cervical VNS or transcutaneous stimulation of the auricular branch of the vagus, and serum cytokines were measured. Transcutaneous stimulation resulted in decreased serum levels of TNF- $\alpha$, IL- $1 \beta$, and 6 versus sham-stimulated animals, although not as robustly as when direct VNS was administered. In our hands, transcutaneous stimulation of the auricular branch of the vagus nerve modulated serum TNF in endotoxemic rodents, although the effect was inconsistent between experiments (Y Levine, unpubl.). A different group has developed a stimulator that uses a composite of high- and low-frequency stimulation to penetrate deeply in the skin of the neck, and have reported serum TNF- $\alpha$ modulation in healthy human subjects, although the change in TNF- $\alpha$ was extremely small (Brock et al. 2017). They have published ample indirect evidence that brain centers associated with cervical or auricular VNS are activated by this therapy (Simon and Blake 2017), but have yet to directly show evoked potentials on the cervical vagus nerve. In our hands, transcutaneous stimulation using their published parameters at $1 \mathrm{~Hz}$ through the shaved neck of an anesthetized rat did not result in evoked potentials on the cervical vagus. In contrast, when the same stimulation is applied directly to the nerve through a cuff electrode, robust evoked potentials were detected (Y Levine, unpubl.).

Groups have investigated minimally invasive means of activating the cervical vagus nerve, potentially enabling activation of the inflammatory reflex in acute indications, such as immediately following stroke or myocardial infarction, where a permanent implant would not be appropriate. Terrando and colleagues have recently shown protection against detrimental central effects of LPS administration in mice by stimulating the cervical vagus nerve through a percutaneous needle electrode placed under ultrasound guidance (Huffman et al. 2019). With a focus on chronic disease, we identified several additional intervention points that grant access to the vagus nerve with minimal invasiveness, potentially allowing for permanent fixation. Alternatively, an acute trial stimulation could be performed using biomarkers to dictate whether a permanent chronic stimulator on the vagus nerve should be implanted. We tailored electrodes to evaluate additional intervention points in canine models to assess the feasibility and reliability of these alternative stimulation locations. The animals were chronically implanted with one of four different types of electrodes, including a bipolar nerve cuff placed directly on the vagus nerve and a linear multichannel field effect electrode array placed adjacent to the vagus within the carotid sheath. To assure that the linear arrays would not shift before tissue encapsulation, sutures were added to stabilize the arrays in position. In addition, two intravascular leads were placed at junctures directly opposed to typical vagus nerve positions: a multichannel helical 
electrode in the superior vena cava (SVC) and a helical pacing lead in the internal jugular vein (IJV). Because of the large size of the SVC, an eight-channel helical array was constructed, which expanded against the walls of the SVC, and C-arm fluoroscopy was used to position the array. After the animals recovered from surgery for at least 14 days, nonsedated conscious stimulation sessions took place once weekly through percutaneous leads with stimulation current output increased from the previous session until discomfort was observed. Change from prestimulation baseline of LPS-induced TNF production in an ex vivo bioassay was the biomarker used to determine activation of the inflammatory reflex. We observed that only stimulation delivered directly to the nerve via cuff electrode consistently resulted in a reduction in TNF $>25 \%$ from baseline in all animals, as seen in Table 1 (M Faltys, unpubl.). Only one in four canines had reduced bioassay TNF following stimulation through a proximity electrode placed inside the carotid sheath. Taken together, these data indicated that a cuff electrode directly on the vagus nerve is the best way to reliably evoke the inflammatory reflex.

\section{EVOLVING TO A MICROSTIMULATOR}

Traditional cuff and implantable pulse generators (IPGs) are not well-suited for VNS and, particularly, for applying VNS to treat the indications that SetPoint is addressing, including chronic inflammatory disorders such as RA. For example, the Livanova VNS system uses the Huntington Helix electrode on the vagus nerve in the neck, and connects with an IPG

Table 1. Investigated intervention points in canine

\begin{tabular}{llc}
\hline \multicolumn{2}{c}{$\begin{array}{c}\text { Electrode } \\
\text { type }\end{array}$} & \multicolumn{1}{c}{ Results } \\
\hline IJV & Intravascular & No biomarker modulation \\
SVC & Intravascular & No biomarker modulation \\
Carotid & Proximity & $25 \%$ showed biomarker \\
sheath & & modulation \\
Cervical & Cuff & $100 \%$ showed biomarker \\
vagus & & modulation \\
\hline
\end{tabular}

IJV, Internal jugular vein; SVC, superior vena cava. implanted in the patient's chest (Amar et al. 1998). Conventional leads are delicate structures that are made from fine wire and silicone rubber, yet must withstand continuous flexing, especially in the neck. A viable lead may take years of design and surgical technique iterations in humans to achieve the levels of reliability now expected or required for implantable medical devices. Patients may be willing to have a lifesustaining pacemaker or defibrillator, or an epilepsy stimulation device, implanted, particularly when the patient has no or very limited alternative treatment options. In contrast, implantable device treatments for the treatment of chronic inflammatory disease may be met with skepticism by both patients and their physicians, who may be uncomfortable with the prospect of a neurovascular surgery with multiple incisions and visible scars. Another perceived disadvantage may include significant internal scar tissue buildup around a tethered lead and cuff (Espinosa et al. 1999). An implanted lead body gets pulled and tugged every time the patient moves their neck, resulting in continued formation of a thick fibrotic sheath around the device until all the stresses are neutralized.

These and other limitations of leads were recognized decades ago. In 1988, Loeb proposed the feasibility of designing millimeter- and submillimeter-scale neurostimulators made possible by the coupling of radio frequency (RF) coils to transmit power, eliminating the need for bulky batteries and associated circuitry (Heetderks 1988). Schulman, Loeb, and Troyk subsequently developed an injectable hermetically sealed glass microstimulator, which was powered and controlled by an external RF coil (Loeb et al. 1991). This device came to be called the RF BION (Schulman et al. 2008). The RF BION was designed to be injected into muscle in proximity to a nerve, and activate the tissue by passing current between the two electrodes on opposite sides of the device. The Alfred E. Mann Foundation (AMF) continued to develop the RF BION and greatly increased the reliability of the device by using a ceramic rather than glass hermetic housing, and deployed the device in several clinical trials (Dupont et al. 2001; Buller et al. 2002; Richmond et al. 2003; Misawa et al. 2004). 
Y.A. Levine et al.

Although the RF BION eliminated leads, it instead required the patient to apply a coil to the body whenever stimulation was required. To solve this issue, the idea of a battery-powered BION was conceived by researchers at AMF who worked with the Jet Propulsion Laboratory (JPL) to develop a small, cylindrical lithium ion rechargeable battery that was later manufactured by a company, Quallion, formed specifically to produce this battery for medical use (Schulman et al. 2008). The resulting battery was a highly reliable cell that can be discharged completely, down to zero volts, and later fully recharged. This so-called zero-volt technology was an important advance because it eliminated the need to remove the device from the patient's body if the patient did not recharge their device for a prolonged period. Advanced Bionics developed and used the battery-powered BION technology, and implanted patients for the treatment of urinary incontinence and migraine headaches (Schulman et al. 2008). SetPoint adopted the idea of powering the implant with the third generation of the Quallion battery so that a patient does not need to worry about when to dose themselves, allowing the SetPoint implant to consistently deliver stimulation without concerns about patient compliance.

Injectable microstimulators like the BION devices are not suitable for modulating the inflammatory reflex, in part because they include proximity electrodes that would not reliably trigger the inflammatory response, as we have reported in the canine experiment shown in Table 1. SetPoint Medical developed the concept of an integrated IPG cuff electrode to overcome this issue. The integrated cuff was possible to develop because the SetPoint IPG technology was sufficiently small such that the entire IPG device could be encapsulated within a silicone cuff alongside the nerve. Early in the development of the SetPoint device, a preliminary model of this integrated cuff was presented to a group of neuro- and vascular surgeons at a preclinical facility, which led to SetPoint adding a groove on a new "MicroRegulator" device, within which contacts are embedded. The entire assembly could then be situated inside a silicone support, called a positioning and orientation device (POD). The
POD was derived from the conceptual model of a pea in a pod, and it positions and orients the electrodes against the nerve and insulates the electrical path to more directly and efficiently route the current through the nerve. To assure that the MicroRegulator remains in place, a suture is used to tie the POD closed (Fig. 3).

Although the implantable battery greatly relieved the patient's manual dosing responsibilities, the implantable battery still required the patient to recharge it every week. This may be accomplished by wearing a wireless charging band that interacts with the MicroRegulator's onboard telemetry. The charging band contains a coil that is also used to recharge the device battery by RF induction. One challenge of recharging batteries is to manage the heat so as not to damage anatomical structures near the implant. For example, ISO14708 states that the temperature of an implantable device shall not increase more than $2^{\circ} \mathrm{C}$ even in the case of a single failure. Because inductive charging systems are not perfectly efficient, heat is generated when charging energy is received from the external coil. When the SetPoint system is operating normally, external energy is regulated to the coil and the temperature of the device is kept within limits. However, in the case in which the external charger malfunctions and too much energy is transmitted by the external coil, an integrated temperature sensor reduces MicroRegulator energy absorption so that the device does not overheat.

It may be valuable to measure the effect of stimulation using radiologically acquired biomarkers to objectively evaluate the clinical efficacy of the device in treating the disease. For example, magnetic resonance imaging (MRI) has become an increasing important marker for RA progression (Ostergaard et al. 2017). A primary goal of the SetPoint MicroRegulator was ensuring that the device was clinically compatible with MRI. MRI use has been a problem for many other implantable medical devices, particularly those with leads, because typical leads can act like an antenna and absorb the energy created by the dynamic RF field of the MRI device when operating at the Larmor frequency (Gupte et al. 2011). The Larmor frequency is dependent on the static magnetic field 


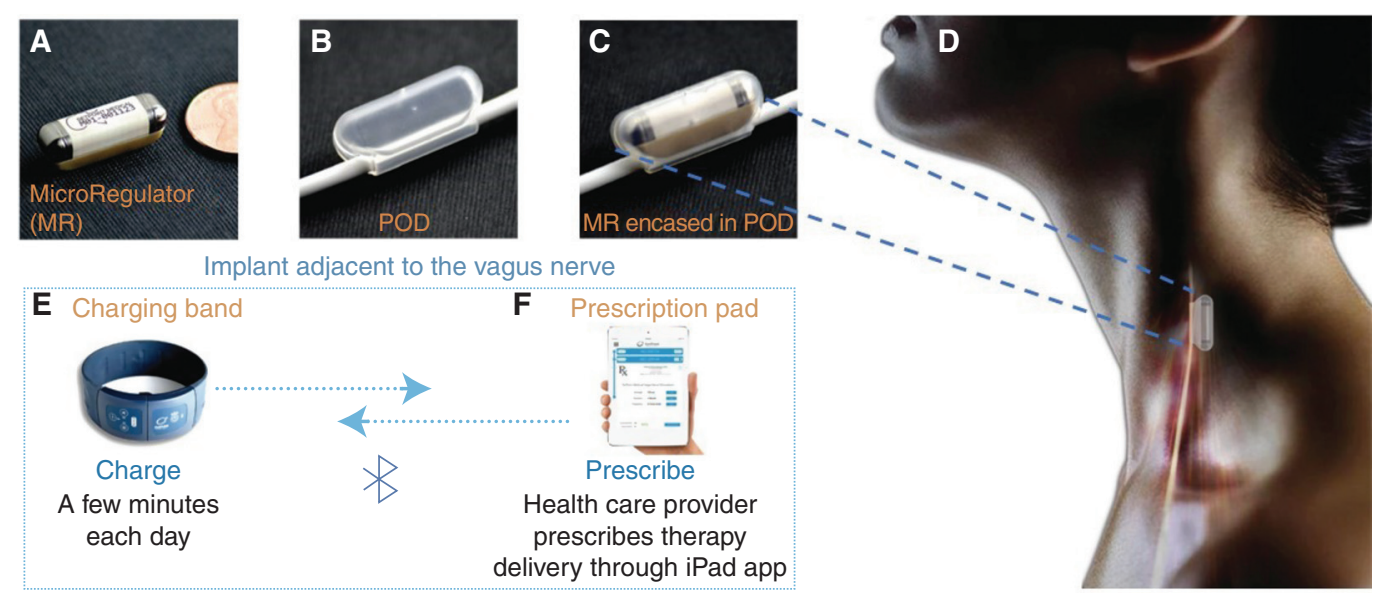

Figure 3. Schematic representation of MicroRegulator (MR) system. The MR $(A)$ is designed to be fully selfcontained, including the application-specific integrated circuit (ASIC), integrated electrodes, a rechargeable battery, and a near-field antenna used both for telemetry and battery recharging. The positioning and orientation device $(\mathrm{POD})(B, C)$ encases the $\mathrm{MR}$, ensuring close contact with the nerve $(D)$. The charging band $(E)$ contains a radio frequency $(\mathrm{RF})$ coil and is used to recharge the device battery and program-independent MR function. The charging band communicates with the prescription pad $(F)$, an iPad-based control application enabling the health care provider to prescribe doses on the implanted MR.

strength. This RF energy would be dissipated into both ends of the lead, tissue, and device, potentially damaging one or more of these. Small implants without extended leads have an inherent advantage, as they are very poor RF antennas because of their short length relative to the MRI wavelength (Shellock et al. 2004; Powell et al. 2013). An optimal antenna to receive energy is approximately between $1 / 4$ and 1 wavelength, wherein a $1 / 4$ wavelength of 66 $\mathrm{MHz}$ is $113 \mathrm{~cm}$. In contrast, the SetPoint MicroRegulator is only $2 \mathrm{~cm}$ long.

Hermetic packaging of electronics has been proven to be necessary for long-term reliability (Thomas 1976). However, hermetic packages are not perfect and, in particular, small devices may be more vulnerable to penetrating fluid. It must be assumed that package leak rates are only as good as the test, before three monolayers of water condense on electronic surfaces and cause a conduction path. We extended the expected implanted life of the SetPoint MR devices by adding a "getter" material that absorbs excess moisture to increase the life of the device (Loeb and Richmond 2000). Another technology that we incorporated was wafer-scale ultralow power microprocessors, allowing the stimulator to be programmable and adaptable to the latest advancements in bioelectronic medicine by downloading new software.

The idea that modulating the inflammatory reflex is analogous to administering a drug is a paradigm shift from classic neuromodulation applications. Rather than stimulating a patient at a particular duty cycle, periodic doses can be delivered on a time schedule, using a real-time clock. Thus, the electrical treatment "doses" are akin to the delivery of pharmaceutical drug doses. Although rheumatologists and other physicians that treat patients with inflammatory diseases do not typically have experience with active implantable medical devices or neural stimulation, care was taken to extend their treatment domain by presenting an electronic prescription pad to control the MicroRegulator. The physician writes a prescription that gets translated to neural stimulation parameters.

\section{FIRST-IN-HUMAN AND BEYOND}

Harnessing the inflammatory reflex for the treatment of inflammation-mediated diseases 
Y.A. Levine et al.

is a concept full of promise and opportunity. The fundamental discovery of this prototypic innate neuroimmune reflex was followed by progressive exploration of the therapeutic space, focused on biomarker-driven optimization of stimulation parameters and next-generation stimulator platforms. Through application of these lessons, we ran a successful proof-of-concept study in humans, and built a fundamentally different implanted neurostimulator system. The MicroRegulator system is currently undergoing a U.S.based first-in-human, double-blind safety study in 14 subjects with multiple biologic and targeted disease-modifying antirheumatic drugs (DMARD) refractory RA. Following the completion of the first-in-human study, we are looking forward to a pivotal study and, if successful, the addition of this pioneering bioelectronic medicine to the physician's armamentarium.

\section{REFERENCES}

Amar AP, Heck CN, Levy ML, Smith T, DeGiorgio CM, Oviedo S, Apuzzo ML. 1998. An institutional experience with cervical vagus nerve trunk stimulation for medically refractory epilepsy: Rationale, technique, and outcome. Neurosurgery 43: 1265-1276. discussion 1276-1280.

Andersson U, Tracey KJ. 2012a. Neural reflexes in inflammation and immunity. J Exp Med 209: 1057-1068. doi:10 .1084/jem.20120571

Andersson U, Tracey KJ. 2012b. Reflex principles of immunological homeostasis. Annu Rev Immunol 30: 313-335. doi:10.1146/annurev-immunol-020711-075015

Ardell JL, Nier H, Hammer M, Southerland EM, Ardell CL, Beaumont E, KenKnight BH, Armour JA. 2017. Defining the neural fulcrum for chronic vagus nerve stimulation: Implications for integrated cardiac control. J Physiol 595: 6887-6903. doi:10.1113/JP274678

Biomarkers Definitions Working Group. 2001. Biomarkers and surrogate endpoints: Preferred definitions and conceptual framework. Clin Pharmacol Ther 69: 89-95. doi: 10.1067/mcp.2001.113989

Bonaz B, Sinniger V, Hoffmann D, Clarencon D, Mathieu N, Dantzer C, Vercueil L, Picq C, Trocme C, Faure P, et al. 2016. Chronic vagus nerve stimulation in Crohn's disease: A 6-month follow-up pilot study. Neurogastroenterol Motil 28: 948-953. doi:10.1111/nmo.12792

Borovikova LV, Ivanova S, Zhang M, Yang H, Botchkina GI Watkins LR, Wang H, Abumrad N, Eaton JW, Tracey KJ. 2000. Vagus nerve stimulation attenuates the systemic inflammatory response to endotoxin. Nature 405: 458462. doi:10.1038/35013070

Brock C, Brock B, Aziz Q, Møller HJ, Pfeiffer Jensen M Drewes AM, Farmer AD. 2017. Transcutaneous cervical vagal nerve stimulation modulates cardiac vagal tone and tumor necrosis factor- $\alpha$. Neurogastroenterol Motil 29: e12999. doi:10.1111/nmo.12999

Buller J, Cundiff G, Noel K, Van Rooyen J, Leffler K, Ellerkman M, Bent A. 2002. RF BIONTM: An injectable microstimulator for the treatment of overactive bladder disorders in adult females. Eur Urol Suppl 1: 40. doi:10 .1016/S1569-9056(02)80149-X

Castoro MA, Yoo PB, Hincapie JG, Hamann JJ, Ruble SB, Wolf PD, Grill WM. 2011. Excitation properties of the right cervical vagus nerve in adult dogs. Exp Neurol 227: 62-68. doi:10.1016/j.expneurol.2010.09.011

Costantini TW, Bansal V, Peterson CY, Loomis WH, Putnam JG, Rankin F, Wolf P, Eliceiri BP, Baird A, Coimbra R. 2010. Efferent vagal nerve stimulation attenuates gut barrier injury after burn: Modulation of intestinal occludin expression. J Trauma 68: 1349-1354; discussion 1354-1346. doi:10.1097/TA.0b013e3181dccea0

Dalli J, Colas RA, Arnardottir H, Serhan CN. 2017. Vagal regulation of group 3 innate lymphoid cells and the immunoresolvent PCTR1 controls infection resolution. Immunity 46: 92-105. doi:10.1016/j.immuni.2016.12.009

De Ferrari GM, Crijns HJ, Borggrefe M, Milasinovic G, Smid J, Zabel M, Gavazzi A, Sanzo A, Dennert R, Kuschyk J, et al. 2011. Chronic vagus nerve stimulation: A new and promising therapeutic approach for chronic heart failure. Eur Heart J 32: 847-855. doi:10.1093/eurheartj/ehq391

Dupont A, Bagg SD, Creasy JL, Romano C, Romano D, Loeb GE, Richmond FJR. 2001. Clinical trials of BION injectable neuromuscular stimulators. Proc IFESS 6: 7-9.

Espinosa J, Aiello MT, Naritoku DK. 1999. Revision and removal of stimulating electrodes following long-term therapy with the vagus nerve stimulator. Surg Neurol 51: 659-664. doi:10.1016/S0090-3019(99)00046-4

Evrengul H. 2004. Heart rate variability in patients with rheumatoid arthritis. Rheumatol Int 24: 198-202. doi: 10.1007/s00296-003-0357-5

Gao XY, Zhang SP, Zhu B, Zhang HQ. 2008. Investigation of specificity of auricular acupuncture points in regulation of autonomic function in anesthetized rats. Auton Neurosci 138: 50-56. doi:10.1016/j.autneu.2007.10.003

Gold MR, Van Veldhuisen DJ, Hauptman PJ, Borggrefe M, Kubo SH, Lieberman RA, Milasinovic G, Berman BJ, Djordjevic S, Neelagaru S, et al. 2016. Vagus nerve stimulation for the treatment of heart failure: The INOVATEHF Trial. J Am Coll Cardiol 68: 149-158. doi:10.1016/j .jacc.2016.03.525

Gupte AA, Shrivastava D, Spaniol MA, Abosch A. 2011. MRI-related heating near deep brain stimulation electrodes: More data are needed. Stereotact Funct Neurosurg 89: 131-140. doi:10.1159/000324906

Handforth A, DeGiorgio CM, Schachter SC, Uthman BM, Naritoku DK, Tecoma ES, Henry TR, Collins SD, Vaughn BV, Gilmartin RC, et al. 1998. Vagus nerve stimulation therapy for partial-onset seizures: A randomized active-control trial. Neurology 51: 48-55. doi:10.1212/ WNL.51.1.48

He W, Fong Y, Marano MA, Gershenwald JE, Yurt RW, Moldawer LL, Lowry SF. 1992. Tolerance to endotoxin prevents mortality in infected thermal injury: Association with attenuated cytokine responses. J Infect Dis 165: 859864. doi:10.1093/infdis/165.5.859 
Heck C, Helmers SL, DeGiorgio CM. 2002. Vagus nerve stimulation therapy, epilepsy, and device parameters: Scientific basis and recommendations for use. Neurology 59: S31-S37. doi:10.1212/WNL.59.6_suppl_4.S31

Heetderks WJ. 1988. RF powering of millimeter- and submillimeter-sized neural prosthetic implants. IEEE Trans Biomed Eng 35: 323-327. doi:10.1109/10.1388

Hoffman HH, Schnitzlein HN. 1961. The numbers of nerve fibers in the vagus nerve of man. Anat Rec 139: 429-435. doi:10.1002/ar.1091390312

Huffman WJ, Subramaniyan S, Rodriguiz RM, Wetsel WC, Grill WM, Terrando N. 2019. Modulation of neuroinflammation and memory dysfunction using percutaneous vagus nerve stimulation in mice. Brain Stimul 12: 19-29. doi:10.1016/j.brs.2018.10.005

Huston JM, Ochani M, Rosas-Ballina M, Liao H, Ochani K, Pavlov VA, Gallowitsch-Puerta M, Ashok M, Czura CJ, Foxwell B, et al. 2006. Splenectomy inactivates the cholinergic antiinflammatory pathway during lethal endotoxemia and polymicrobial sepsis. J Exp Med 203: 1623-1628. doi:10.1084/jem.20052362

Huston JM, Gallowitsch-Puerta M, Ochani M, Ochani K, Yuan R, Rosas-Ballina M, Ashok M, Goldstein RS, Chavan S, Pavlov VA, et al. 2007. Transcutaneous vagus nerve stimulation reduces serum high mobility group box 1 levels and improves survival in murine sepsis. Crit Care Med 35: 2762-2768.

Kees MG, Pongratz G, Kees F, Schölmerich J, Straub RH. 2003. Via $\beta$-adrenoceptors, stimulation of extrasplenic sympathetic nerve fibers inhibits lipopolysaccharide-induced TNF secretion in perfused rat spleen. J Neuroimmunol 145: 77-85. doi:10.1016/j.jneuroim.2003.09.011

Konsman JP, Parnet P, Dantzer R. 2002. Cytokine-induced sickness behaviour: Mechanisms and implications. Trends Neurosci 25: 154-159. doi:10.1016/S0166-2236 (00)02088-9

Koopman FA, Chavan SS, Miljko S, Grazio S, Sokolovic S, Schuurman PR, Mehta AD, Levine YA, Faltys M, Zitnik $\mathrm{R}$, et al. 2016a. Vagus nerve stimulation inhibits cytokine production and attenuates disease severity in rheumatoid arthritis. Proc Natl Acad Sci 113: 8284-8289. doi:10.1073/ pnas. 1605635113

Koopman FA, Tang MW, Vermeij J, de Hair MJ, Choi IY, Vervoordeldonk MJ, Gerlag DM, Karemaker JM, Tak PP. 2016b. Autonomic dysfunction precedes development of rheumatoid arthritis: A prospective cohort study. EBioMedicine 6: 231-237. doi:10.1016/j.ebiom.2016.02 .029

Koopman FA, van Maanen MA, Vervoordeldonk MJ, Tak PP. 2017. Balancing the autonomic nervous system to reduce inflammation in rheumatoid arthritis. J Intern Med 282: 64-75.

Kwan H, Garzoni L, Liu HL, Cao M, Desrochers A, Fecteau G, Burns P, Frasch MG. 2016. Vagus nerve stimulation for treatment of inflammation: Systematic review of animal models and clinical studies. Bioelectron Med 3: $1-6$.

Levine YA, Caravaca A, Faltys M, Arnold A, Zitnik R. 2013. Vagus nerve stimulation provides prolonged and spleenindependent protection against indomethacin-induced intestinal inflammation. Gastroenterology 144: S-67.
Levine YA, Koopman F, Faltys M, Zitnik R, Tak PP. 2014a. Using traditional preclinical models to guide development of an untraditional inflammation therapy: Neurostimulation of the cholinergic anti-inflammatory pathway in rheumatoid arthritis and inflammatory bowel disease. Bioelectron Med 1: 34-43.

Levine YA, Koopman FA, Faltys M, Caravaca A, Bendele A, Zitnik R, Vervoordeldonk MJ, Tak PP. 2014b. Neurostimulation of the cholinergic anti-inflammatory pathway ameliorates disease in rat collagen-induced arthritis. PLoS ONE 9: e104530. doi:10.1371/journal.pone.0104530

Levine YA, Simon JM, Koopman F, Faltys M, Zitnik R, Tak PP. 2017. VNS for treatment of inflammatory joint diseases. In Electroceuticals: Advances in electrostimulation therapies (ed. Majid A), pp. 35-53. Springer, New York.

Levine YA, Faltys M, Zitnik R. 2018a. Activation of the inflammatory reflex in rheumatoid arthritis and inflammatory bowel disease; preclinical evidence. In Neuromodulation, 2nd ed. (ed. Rezai A, et al.), pp. 1493-1502. Elsevier, San Diego.

Levine YA, Simon J, Faltys M. 2018b. Neurophysiologic and chronic safety testing of a miniaturized active implanted device with integrated electrodes for bioelectronic medicine applications. Conf Proc IEEE Eng Med Biol Soc 2018: 3689-3692.

Li P, Liu H, Sun P, Wang X, Wang C, Wang L, Wang T. 2016. Chronic vagus nerve stimulation attenuates vascular endothelial impairments and reduces the inflammatory profile via inhibition of the NF- $\mathrm{\kappa B}$ signaling pathway in ovariectomized rats. Exp Gerontol 74: 43-55. doi:10.1016/ j.exger.2015.12.005

Loeb GE, Richmond FJR. 2000. BION ${ }^{\mathrm{TM}}$ implants for therapeutic and functional electrical stimulation. In Neural prosthesis for restoration of sensory and motor function (ed. Moxon KA, Chapin JK). CRC Press, Boca Raton, FL.

Loeb GE, Zamin CJ, Schulman JH, Troyk PR. 1991. Injectable microstimulator for functional electrical stimulation. Med Biol Eng Comput 29: NS13-NS19.

Lu B, Kwan K, Levine YA, Olofsson PS, Yang H, Li J, Joshi S, Wang H, Andersson U, Chavan SS, et al. 2014. $\alpha 7$ Nicotinic acetylcholine receptor signaling inhibits inflammasome activation by preventing mitochondrial DNA release. Mol Med 20: 350-358.

Matteoli G, Boeckxstaens GE. 2013. The vagal innervation of the gut and immune homeostasis. Gut 62: 1214-1222. doi:10.1136/gutjnl-2012-302550

Matteoli G, Gomez-Pinilla PJ, Nemethova A, Di Giovangiulio M, Cailotto C, van Bree SH, Michel K, Tracey KJ, Schemann M, Boesmans W, et al. 2014. A distinct vagal anti-inflammatory pathway modulates intestinal muscularis resident macrophages independent of the spleen. Gut 63: 938-948. doi:10.1136/gutjnl-2013-304676

McIntyre CC, Grill WM. 1998. Sensitivity analysis of a model of mammalian neural membrane. Biol Cybern 79: 2937. doi:10.1007/s004220050455

Meregnani J, Clarençon D, Vivier M, Peinnequin A, Mouret C, Sinniger V, Picq C, Job A, Canini F, Jacquier-Sarlin M, et al. 2011. Anti-inflammatory effect of vagus nerve stimulation in a rat model of inflammatory bowel disease. Auton Neurosci 160: 82-89. doi:10.1016/j.autneu.2010 .10 .007 
Y.A. Levine et al.

Mirakaj V, Dalli J, Granja T, Rosenberger P, Serhan CN 2014. Vagus nerve controls resolution and pro-resolving mediators of inflammation. J Exp Med 211: 1037-1048. doi:10.1084/jem.20132103

Misawa A, Matsunaga T, Hatakeyama K, Zilberman Y, Cosendai G, Ripley A. 2004. The use of the RF BION ${ }^{\circledR}$ Microstimulator to relieve pain due to shoulder subluxation in chronic hemiplegic stroke patient-A case report. In 9th Annual Conference of the International FES Society September 2004, Bournemouth, UK.

Niijima A. 1996. The afferent discharges from sensors for interleukin $1 \beta$ in the hepatoportal system in the anesthetized rat. J Auton Nerv Syst 61: 287-291. doi:10.1016/ S0165-1838(96)00098-7

Olofsson PS, Katz DA, Rosas-Ballina M, Levine YA, Ochani M, Valdes-Ferrer SI, Pavlov VA, Tracey KJ, Chavan SS 2012a. $\alpha 7$ nicotinic acetylcholine receptor $(\alpha 7 \mathrm{nAChR})$ expression in bone marrow-derived non- $\mathrm{T}$ cells is required for the inflammatory reflex. Mol Med 18: 539543. doi:10.2119/molmed.2011.00405

Olofsson PS, Rosas-Ballina M, Levine YA, Tracey KJ. 2012b. Rethinking inflammation: Neural circuits in the regulation of immunity. Immunol Rev 248: 188-204. doi:10 $.1111 / \mathrm{j} .1600-065 \mathrm{X} .2012 .01138 . \mathrm{x}$

Olofsson PS, Levine YA, Caravaca A, Chavan SC, Pavlov VA, Faltys M, Tracey KJ. 2015. Single-pulse and unidirectional electrical activation of the cervical vagus nerve reduces tumor necrosis factor in endotoxemia. Bioelectron Med 2: 37-42. doi:10.15424/bioelectronmed.2015.00006

Ostergaard M, Peterfy CG, Bird P, Gandjbakhch F, Glinatsi D, Eshed I, Haavardsholm EA, Lillegraven S, Boyesen P, Ejbjerg B, et al. 2017. The OMERACT rheumatoid arthritis magnetic resonance imaging (MRI) scoring system: Updated recommendations by the OMERACT MRI in arthritis working group. J Rheumatol 44: 1706-1712. doi:10.3899/jrheum.161433

Parrish WR, Rosas-Ballina M, Gallowitsch-Puerta M, Ochani M, Ochani K, Yang LH, Hudson L, Lin X, Patel N, Johnson SM, et al. 2008. Modulation of TNF release by choline requires $\alpha 7$ subunit nicotinic acetylcholine receptor-mediated signaling. Mol Med 14: 567-574 doi:10.2119/2008-00079.Parrish

Pavlov VA, Chavan SS, Tracey KJ. 2018. Molecular and functional neuroscience in immunity. Аnпu Rev Immunol 36: 783-812. doi:10.1146/annurev-immunol-042617053158

Powell R, Pless E, Caparso A. 2013. MRI compatibility of the Autonomic Technologies Inc (ATI) SPG neurostimulator-New treatment for cluster headache $(\mathrm{CH})$. J Headache Pain 14: P228. doi:10.1186/1129-2377-14-S1-P228

Richmond FJR, Dupont A, Tran WH, Stein RB, Romano C, Loeb GE. 2003. Tactical application of sensorimotor prosthetic technology. In Proceedings of the 25th Annual International Conference of the IEEE Engineering in Medicine and Biology Society (IEEE Cat No03CH37439), pp. 2017-2020, Vol. 2013.

Rosas-Ballina M, Ochani M, Parrish WR, Ochani K, Harris YT, Huston JM, Chavan S, Tracey KJ. 2008. Splenic nerve is required for cholinergic antiinflammatory pathway control of TNF in endotoxemia. Proc Natl Acad Sci 105: 11008-11013. doi:10.1073/pnas.0803237105
Rosas-Ballina M, Olofsson PS, Ochani M, Valdes-Ferrer SI, Levine YA, Reardon C, Tusche MW, Pavlov VA, Andersson U, Chavan S, et al. 2011. Acetylcholinesynthesizing $\mathrm{T}$ cells relay neural signals in a vagus nerve circuit. Science 334: 98-101. doi:10.1126/science. 120 9985

Sajadieh A, Nielsen OW, Rasmussen V, Hein HO, Abedini S, Hansen JF. 2004. Increased heart rate and reduced heartrate variability are associated with subclinical inflammation in middle-aged and elderly subjects with no apparent heart disease. Eur Heart J 25: 363-370. doi:10.1016/j.ehj .2003.12.003

Schulman J, Mobley JP, Wolfe J, Davis R, Arcos I. 2008. An implantable bionic network of injectable neural prosthetic devices: The future platform for functional electrical stimulation and sensing to restore movement and sensation. In Neuroengineering (ed. DiLorenzo DJ). CRC Press, Boca Raton, FL.

Serhan CN. 2014. Pro-resolving lipid mediators are leads for resolution physiology. Nature 510: 92-101. doi:10.1038/ nature13479

Serhan CN, de la Rosa X, Jouvene CC. 2018. Cutting edge: Human vagus produces specialized proresolving mediators of inflammation with electrical stimulation reducing proinflammatory eicosanoids. J Immunol 201: 31613165. doi:10.4049/jimmunol.1800806

Shafer LL. 2010. Device and method for inhibiting release of pro-inflammatory mediator. U.S. patent no. 7,769, 442.

Shellock FG, Cosendai G, Park SM, Nyenhuis JA. 2004. Implantable microstimulator: Magnetic resonance safety at 1.5 Tesla. Invest Radiol 39: 591-599. doi:10.1097/01 .rli.0000138090.43450.ec

Simon B, Blake J. 2017. Mechanism of action of noninvasive cervical vagus nerve stimulation for the treatment of primary headaches. Am J Manag Care 23: S312-S316.

Stakenborg N, Wolthuis AM, Gomez-Pinilla PJ, Farro G, Di Giovangiulio M, Bosmans G, Labeeuw E, Verhaegen M, Depoortere I, D'Hoore A, et al. 2017. Abdominal vagus nerve stimulation as a new therapeutic approach to prevent postoperative ileus. Neurogastroenterol Motil 29: e13075. doi:10.1111/nmo.13075

Sun P, Zhou K, Wang S, Li P, Chen S, Lin G, Zhao Y, Wang T. 2013. Involvement of MAPK/NF- $K B$ signaling in the activation of the cholinergic anti-inflammatory pathway in experimental colitis by chronic vagus nerve stimulation. PLoS ONE 8: e69424. doi:10.1371/journal.pone .0069424

Tarnawski L, Reardon C, Caravaca AS, Rosas-Ballina M, Tusche MW, Drake AR, Hudson LK, Hanes WM, Li JH, Parrish WR, et al. 2018. Adenylyl cyclase 6 mediates inhibition of TNF in the inflammatory reflex. Front Immunol 9: 2648. doi:10.3389/fimmu.2018.02648

Thomas R. 1976. Moisture, myths, and microcircuits. IEEE Trans Parts Hybrids Packag 12: 167-171. doi:10.1109/ TPHP.1976.1135142

Tracey KJ. 2002. The inflammatory reflex. Nature 420: 853 859. doi:10.1038/nature01321

Vida G, Peña G, Deitch EA, Ulloa L. 2011. $\alpha 7$-cholinergic receptor mediates vagal induction of splenic norepi- 
nephrine. J Immunol 186: 4340-4346. doi:10.4049/jim munol.1003722

Wang H, Yu M, Ochani M, Amella CA, Tanovic M, Susarla S, Li JH, Wang H, Yang H, Ulloa L, et al. 2003. Nicotinic acetylcholine receptor $\alpha 7$ subunit is an essential regulator of inflammation. Nature 421: 384-388. doi:10.1038/na ture01339

Watkins LR, Goehler LE, Relton JK, Tartaglia N, Silbert L, Martin D, Maier SF. 1995. Blockade of interleukin-1 induced hyperthermia by subdiaphragmatic vagotomy: Evidence for vagal mediation of immune-brain commu- nication. Neurosci Lett 183: 27-31. doi:10.1016/03043940(94)11105-R

Yoo PB, Lubock NB, Hincapie JG, Ruble SB, Hamann JJ, Grill WM. 2013. High-resolution measurement of electrically evoked vagus nerve activity in the anesthetized dog. J Neural Eng 10: 026003. doi:10.1088/1741-2560/10/2/026003

Zhao YX, He W, Jing XH, Liu JL, Rong PJ, Ben H, Liu K, Zhu B. 2012. Transcutaneous auricular vagus nerve stimulation protects endotoxemic rat from lipopolysaccharideinduced inflammation. Evid Based Complement Alternat Med 2012: 627023. 


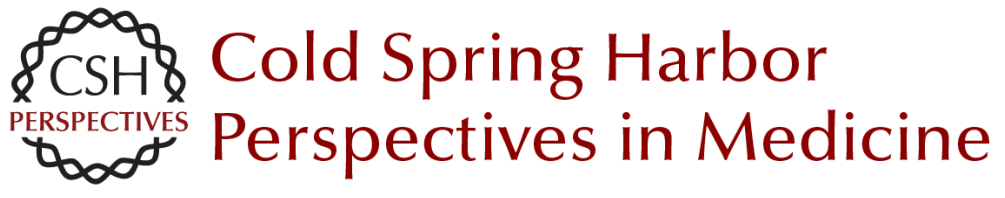

\title{
Harnessing the Inflammatory Reflex for the Treatment of Inflammation-Mediated Diseases
}

\author{
Yaakov A. Levine, Michael Faltys and David Chernoff
}

Cold Spring Harb Perspect Med 2020; doi: 10.1101/cshperspect.a034330 originally published online March 4, 2019

\section{Subject Collection Bioelectronic Medicine}

Neural Control of Inflammation: Bioelectronic Medicine in Treatment of Chronic Inflammatory Disease

Michael Eberhardson, Laura Tarnawski, Monica Centa, et al.

Noninvasive Neuromodulation of Peripheral Nerve Pathways Using Ultrasound and Its Current

Therapeutic Implications

Christopher Puleo and Victoria Cotero

Enteric Neuromodulation for the Gut and Beyond Yogi A. Patel and Pankaj J. Pasricha

Optogenetic Control of the Peripheral Nervous System

Rui B. Chang

Closed-Loop Neuromodulation in Physiological and Translational Research

Stavros Zanos

Electrical Impedance Methods in Neuromuscular Assessment: An Overview

Seward B. Rutkove and Benjamin Sanchez

Optogenetic Medicine: Synthetic Therapeutic

Solutions Precision-Guided by Light

Haifeng Ye and Martin Fussenegger

Technobiology's Enabler: The Magnetoelectric Nanoparticle

Sakhrat Khizroev
Bioelectronic Medicine: From Preclinical Studies on the Inflammatory Reflex to New Approaches in Disease Diagnosis and Treatment

Valentin A. Pavlov, Sangeeta S. Chavan and Kevin

J. Tracey

Vagus Nerve Stimulation and the Cardiovascular System

Michael J. Capilupi, Samantha M. Kerath and

Lance B. Becker

Harnessing the Inflammatory Reflex for the

Treatment of Inflammation-Mediated Diseases

Yaakov A. Levine, Michael Faltys and David Chernoff

Recording and Decoding of Vagal Neural Signals Related to Changes in Physiological Parameters and Biomarkers of Disease Theodoros P. Zanos

Restoring Movement in Paralysis with a

Bioelectronic Neural Bypass Approach: Current

State and Future Directions Chad E. Bouton

Bioelectronic Medicine--Ethical Concerns Samuel Packer, Nicholas Mercado and Anita Haridat

Use of Bioelectronics in the Gastrointestinal Tract Larry Miller, Aydin Farajidavar and Anil Vegesna

Vagus Nerve Stimulation at the Interface of BrainGut Interactions

Bruno Bonaz, Valérie Sinniger and Sonia Pellissier

For additional articles in this collection, see http://perspectivesinmedicine.cshlp.org/cgi/collection/ 